\title{
A smoke screen: youth cannabis abuse may lead to increase in opiates use
}

\author{
Yifrah Kaminer* \\ Departments of Psychiatry and Pediatrics, Alcohol Research Center and Injury Prevention Center, UConn School of Medicine, USA
}

According to the Old Testament danger to the Kingdom of Judea usually came from the North.

This Biblical analogy might be relevant for the state of Connecticut after our neighboring state of Massachusetts (MA) as well as Canada have most recently legalized the recreational use of marijuana over the age of 21 years. This law will get into effect once they will figure out ALL the rules and regulations to be implemented. By then the cities of Boston and Springfield MA, will host approximately 50 and a dozen cannabis sale stores respectively.

How would this new reality affect the health and quality of life of Connecticut youth who would take advantage of increased availability of legal cannabis? Furthermore, what impact might this have on the trend of the opioid abuse epidemic sweeping our state?

Cannabis is not a harmless compound. The changes in cannabis legal status have contributed to the biased perception of youth that marijuana use is safe. This perception has been correlated with an increase in the prevalence of marijuana use, decrease in age of first onset (every day approximately 4000 young Americans use cannabis for the first time) and leads to an increase in impaired driving, cannabis dependence, early onset psychosis, school expulsion, diminished academic performance to name a few consequences. Furthermore, the journey of youth who experiment with opioid painkillers and heroin abuse that might lead to a lethal ending usually starts with marijuana use for mood altering purposes. Marijuana has been clearly identified as a gateway drug for progression to use of other drugs in youth. That explains why some adolescents and young adults were lured by the chase of a "better high" via prescription opiates, which are unfortunately easy to attain. These drugs are stolen from relatives or pharmacies, diverted from legal opiate users or simply purchased in the street.

Copyright: (C2018 Kaminer Y. This is an open-access article distributed under the terms of the Creative Commons Attribution License, which permits unrestricted use, distribution, and reproduction in any medium, provided the original author and source are credited.
Most importantly, a recent independent study conducted in Connecticut by Dr. Swindell from Quantitative Services has received little attention regardless of its important conclusion that there is a relationship between marijuana (MJ) use and the abuse of opioid pain medications and heroin. This study that included a control group is based on data from a total of 6000 Connecticut high-school students over a period of 12 years. MJ users reported 14-fold abuse of pain medications and more than 4 -fold of heroin use respectively than nonMJ users. To put these numbers in proportion according to the CT medical examiner report, 75 youth up to the age of 25 years have died from opioids overdose in 2015. This is most probably a conservative number. Furthermore, the current use of an additional illicit drug is about 5 times higher among MJ users. Dr Swindell's report concludes that "these correlations are about the same in towns across CT, regardless of socio-economic and demographics and have been stable since at least 2008".

I can see short sighted politicians rushing to approve legalization in order to compete with Massachusetts on taxation revenues. This move would be ill advised from a public health perspective given the dire consequences of the legalization experiment in Colorado. Where there is smoke there is fire and our youth, families and communities have reasons for concern. If we would not engage in effective prevention and allow potential changes in the legal status of marijuana be driven by science and facts instead of economic miscalculations of benefit versus negative consequences, we will have to resort later to a more costly damage control mode in terms of human lives and resources as we have been trying to manage the opioid epidemic with a limited success.
*Correspondence to: Yifrah Kaminer MD, Professor of Psychiatry and Pediatrics, Alcohol Research Center and Injury Prevention Center, UConn School of Medicine, USA, Tel: 860679 4344; E-mail: Kaminer@uchc.edu

Received: November 22, 2018; Accepted: December 04, 2018; Published: December 07, 2018 